\title{
Nevo sebáceo de Jadassohn
}

\author{
Patricio Llancapi Y.': Oriana Paiva $\mathbf{M}^{2}$
}

\author{
Sebaceous nevus of Jadassohn
}

Two pot e-1s with sebcceoss revus of Jadossohn are oreseriled. Th's les on is cn homartoma with a well cicunscribed alopecic picque stape, whose ciameler varies from less than one to a few $\mathrm{cm}$. It is coused by berign hyperplasia of sebaceous glonds. Most common location is on the scalp and less frequenty on the face, trunk ond extremities. n ariy localion, the noturat history is the some, with a -ight risk of ranshomation to matignant neoplosia. The lesion moy oppear in rewtome or rifonts. When potients get lo puberly, it noy develop inio differert kinds of tumours. Treatment is surgizol excis on along peputeral age.

(Key words: r.evus, sevoceoss, jcdassohn.,

El nevo scbáceo de Jadassohn es una lesión hamartoidea en forma de placa alopécica bien circunscrita de unos pocos milímetros a varios centímetros de diámetro, que corresponde a hiperplasia benigna de las glándulas sebáceas. Se localiza generalmente en el cuero cabelludo y más raramente en la cara, el tronco y las extremidades. En cualquier ubicación la historia natural es la misma. Puede presentarse al nacer o durante la inlancia. Al alcanzar la pubertad pueden desarrollarse. a partir de él, tumores de diversos tipos. El tratamiento es la extirpación quirúrgica en la etapa prepuberal. En esta presentación se revisa el tema, a propósito de dos casos clínicos, con el objeto de promover el conocimiento y la identificación oportuna de estos pacientes.

\section{Casos clínicos}

1. Niña de 7 años de edad con una lesión solevantada Jel cuero sabelludo, cuyo tamaño fue aumentando en los ülimos arios. En la zona parietoncepital se observa una lesión en placa. lovemente solevantada de superficie mamelonada, de color rosado pálido de aproximadamente $1.5 \times 2.6 \mathrm{cu}$, sin cabello sobre su superficie (figura I).

2. Varón de 14 años de edad, sin antecedentes personales ni familiares, que sutria desde el nacimiento una lesión

I. Hospiral Clinico Regional de Concepción.

2. Alumna de Cafrera de Medicina, Facultad de Medicina, Universidad de Concepción. en vertek de cuero cabelludo, la que, en los últimos meses, habia sangrado y causado dolor al peinarse. La lesion sobresatfa del vivel del cuero cabelludo. era de color amarillo anaranjado de $2,5 \times 1,5 \mathrm{~cm}$ de diánetro, superficie verrucosa. sin crecimiento de pelo sobre su superficic $y$. er su ángulo inferior, mostraba una pequeña erosión (figura 2).

En ambos casos se readizó la extirpación y cierte primario y no se cncontró signos de malignidad en ninguno de ellos.

\section{Comentario}

El tćmino nevo sebáceo fue usado por primera vez en 1895 por Jadassohn', médico alemán, para describir una hiperplasia de glándulas sebaceas. En 1932 fue introducido por Robinson en la literatura médica americana ${ }^{2}$. Se incluye dentro de las facomatosis y corresponde a una hiperplasia benigna circunscrita que compromete predominantemente a glándulas sebáceas y se acompaña de crecimiento anormal o deficiente de otras estructuras de la piel (glándulas ecrinas, apocrinas, folículos pilosos, epidermis). Puede presentarse al nacer o aparecer en la infancia temprana. Su causa desconocida, aunque se ha postulado una mulación somática o mosaicismo de un gen letal como posible origen. No se conoce su real prevalencia, aunque se considera común de ver en niños. Alper y cols. en 1979 desctibieron nevo sebáceo en $0.3 \%$ de un total de 18 I55 recién nacidos, sin diferencias por sexo. Su importancia radica en que presen- 


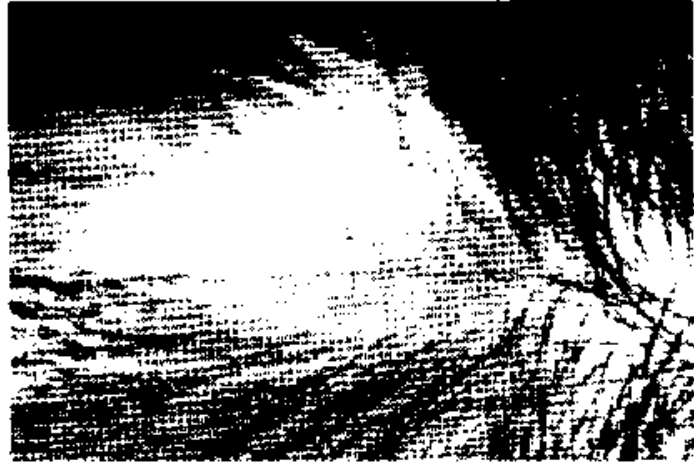

Figura 1: Nevo sebáceo de Jadassohn. Aspecto macroscópico antes de la puberad. Caso 1.

ta un alto riesgo de degeneración carcinoma$\operatorname{tos}^{3 \cdot 8}$

Su localización más común es en el cuero cabelludo $(72 \%)$, aunque puede afectar también la cara $(23 \%)$, el tronco o las extremidades. Generalmente aparece como una placa solitaria ovalada o lineal sin pelo, entre 1 y $10 \mathrm{~cm}$ de diámetro, de superficie aterciopelada. En el recién nacido y hasta la pubertad es de color pálido y superficie verrucosa $a^{3,9}$. Después de la pubertad se torna amarilla, con superficie sebácea verrucosa. Más tarde hay cambios secundarios con crecimiento tumoral sobre la placa original. pudiendo aparecer nódulos y hacerse papilomatosa ${ }^{9}$.

Histologicamente la epidermis aparece hiperqueratótica y papilomatosa. La demis superficial muestra cierto grado de proliferación de tejido fibrovascular con folículos pilosos atróficos, formando filamentos cortos y estrechos de células basales, algunos conectados a la epiciermis. Hasta la pubertad las glándulas sebáceas se encuentran unidas a los folículos pilosos atrófi$\cos$, haciéndose muy hipertroficas en la pubertad y constituyendo el principal componente del nevo. En la profundidad de la dermis hay importante proliferación de glándulas sudoríparas apocrinas y ecrinas.

E1 diagnóstico usualmente es clínico y la lesión debe diferenciarse, en pacientes jovenes (si es pequeño), con el xantogranuloma juvenil, la mastocitosis solitaria y nevo de $\$$ pitz; en pacientes púberes y ancianos puede confundirse con tumores anexiales, xantomas y neoplasias malignas. Si es muy vertucoso, con el nevo epidérmico verrucoso, la verruga vulgar y el li-

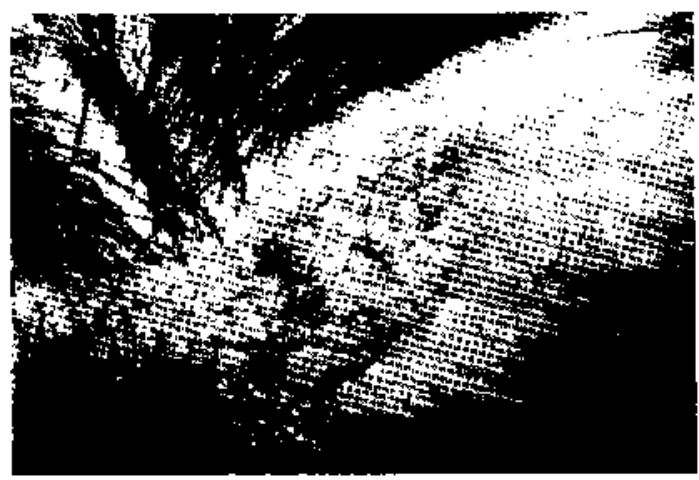

Figura 2: Nevo sebáceo de Jadassohn. Aspecto macroscópico en un paciente púber. Caso 2.

quen simple crónico. Se ha reportado, en pacientes homosexuales infectados con virus de inmunodeficiencia humana, moluscos contagiosos cuyo aspecto era muy similar al nevo sebáceo ${ }^{5}$.

Esta enfermedad puede incluir una variedad de alteraciones en tejidos de origen ectodérmico y mesodérmico y puede simular otras facomatosis como la esclerosis tuberosa, sindrome de Siurge-Weber e incontinencia pigmentaria ${ }^{10}$. En 1962 Feuerstein y Mins establecieron un síndrome neurocutáneo consistente en la triada de nevo sebáceo lineal, epilepsia y retardo mental "'. En 1968 Solomon y colaboraơores comunicaron alta incidencia de anormalidades congénitas en el sistema nervioso central (epilepsia. retardo mental, hidrocefalia), esqueleto (alteraciones en la columna, clavícula y pelvis) y ojo (hemangiomas, opacidad corneal, colobomas) en pacientes con nevo de Jadassohn ${ }^{12}$. Se han demostrado cambios fenotípicos considerables en el lugar de la lesión y a distancia, incluyendo múltiples tumores benignos o malignos raros con manifestaciones inusuales (hepatomegalia, quistes viscerales, vasculopatía, etc. ${ }^{5}$. En 1992 se comunicó un caso de nevo sebáceo de Jadassohn asociado a glioma óptico ${ }^{12}$ y otro en 1993 a lipomatosis mediastinal ${ }^{4}$.

La evolución del nevo de Jadassohn se puede describir en tres etapas: en la infancia y adolescencia, como se ilustra con la niña de esta serie. cuando la glándula sebácea no es prominente y las características del nevo están ausentes, con posibilidad de aparecer vertucosidad en la epidermis, proliferación de tejido fibrovascular, atrofia de folículos pilosos; en la pubertad, en 
que hay gran crecimiento de glándulas sebáceas y cambio de color como en el segundo de nuestros casos y. finalmente, un tercer estado más tardio, en el que puede desarrollarse neoplasia secundaria en aproximadamente el 20 a $30 \%$ de Ios casos ${ }^{3}$. El riesgo de degeneración maligna es más frecuente después de la pubertad, cuando el tratamiento en edad temprana ha sido inadecuado. El tumor más frccuente es el carcinoma basocelular, aunque se han informado casos de carcinomas cscamosos, epiteliomas sebáceos, carcinomas indiferenciados 12 y también siringocistoadenomas (en aproximadamente $10 \%$ de las lesiones) ${ }^{13}$, cistoadenomas apocrinos, queratoacantomas y otros tumores anexiales benignos (trichilemoma, hidradenoma nodular, siringoma condroide) que clínicamente sugerían el diagnóstico de epietelioma basocelular ${ }^{14}$. La extirpación quirúrgica total es curativa, preferiblemente antes de la pubertad, cuando aún no hay crecimiento de glándulas sebáceas.

\section{Resumen}

Se describen dos pacientes con adenoma sebáce o de Jadassohn: una niña de sicte años con una lesión solevantada, pálida y sin pelos en el cuero cabelludo, y un varón de 14 años y una lesión solevantada amarillenta, de aspecto sebáceo, que ilustran las dos primeras etapas, respectivamente, de este tipo de nevo. Estas lesiones deber ser identificadas y extirpadas antes de la pubertad por el riesgo ( 20 a $30 \%$ ) de dege. neración maligna a edad posterior. en la tercerá elapa evolutiva.

(Palabras clave: nevo sebáceo, Jadassohn.)

\section{Referencias}

1. Jadussohn $d:$ Betnerkurgen zur histologie des systematisierten naevi und uber Talgdrusen Naevi. Arch Dermat U Syph $198.5: 3.3: 355-3,56$
2. Robinson $S$ : Nevus sebaccous (Jadassohn). Arch Derm Syph 1932: 26: 663-670.

3. Rahbari H: Organoid nevus (nevus sebaceous). In: Demis J, Clinical Dermatology, Lippincott Company, Philadelphia 1994: 4: 23-24.

4. Tabouda E. Moledo E, Alwarez A. Comellas M: Sebaceous nevus of Jadassohn and primary medialinal lipomatosis. Br J Plast Surg (Scotland) 199.3; 46: 264265.

5. Iin P. Gilli L: Molkuseum contagiosum mimicking sebaceous nevus of Jadassohn, ecthyma an giant condylomata acuminata in HIV-infected patients. Lermarology 1994: 189: 396-398.

6. Comter A, Bryan $H$ : Nevus sebaceous of Jadassobn. Am J Dis Child 1967; ! 14: 23-24,

7 Kotrseff 8G: Hypothesis: Jadassoho nevus phacomatosis: a paracrinopathy with variable phenotype. Am J Med Genet 1992: 43: 651-661.

8 Alper $J$, Holmes $L$, Mihm $M$ : Birthmarks with serious medical significance: nevocellular nevi, sebaceous nevi and multiple cafe au lair spots. J Pediatr 199.5; 5: $697-700$

9. Meneghello $d$, Funtu E. Paris E. Rossetor J: Pediatria (Santiago) 1991: 1607.

10. Janksmira E. Sobaniec W, Czentinkka C, Sobaniec M. Drozdun:ski $W$ : A case of epidermal nevus (Jadassohn's phacomatosis) with changes in the nervous system. Neurol Neurochir Pol 1991: 25; 491496.

11. Solw $K$. Kubuto $T$. Kitai $R$ : Linear sebaceous nevus syndrome (sebaceous nevus of Jadassohn) associated with abnormal neuronal inigration and optic glioma: case report. Neurosurgery 1994; 35: 318-320.

12. Constant E. Daris $D$. The premalignant nature of the sebaceous nevus of Jadassohn. Plastic \& Reconstructive Surgery 1972; 50: 257-259

13. Amold $H$, Odom $R$, James $W$ : Andrew5: Tratado de Dermátología, cóciones cjentíficas y técnicas S.A. Massom-Salvat Medicina 1993;818

14. Bonvalet D, Barrandon $Y_{1}$ Foix Ch, Civarte J: Tumeurs annexielles benignes de survenue tardive sur nàvus vertuco-sebacè (Jadassohn). A propos de 7 cas. Ann Dermatol Venereol 1983; 1 10: 337-342.

15. Larroue $G$, Cattatoube $D$. Ndiaye $B$, Jowen $F$ : Le nevus epidermique verruqueux de la face. Ann Chir Plası Esthet (France) 1991: 36: 160-172.

16. Fitzatrick $T$, Eisen A, Wolff $K$ : Dermacología en medicina general, Editorjal Médica Panamericana 1988: 2256-2257.

17. Rook, Wilkinson. Ebling: Texthook of dermatology. Blackwell Scientific Publication 1992; 1: 456-457. 